\title{
Las mutualidades en Euskadi como entidades de la economía social ${ }^{\&}$
}

\author{
Mutual benefit societies in the Basque Country \\ as entities of the Social Economy
}

Francisco Javier Arrieta Idiakez ${ }^{* 1}$, Gonzalo Martínez Etxeberria ${ }^{2}$, Josune López RodRíguez ${ }^{3}$

${ }^{1}$ Profesor titular de Derecho del Trabajo y de la Seguridad Social de la Universidad de Deusto (javier.arrieta@deusto.es)

2 Profesor doctor de Derecho Constitucional de la Universidad de Deusto

(Gonzalo.metxeberria@deusto.es)

3 Profesora doctora de Derecho Social de la Universidad de Deusto

(josunelopezrodriguez@deusto.es)

Resumen: A través de este estudio se pretenden identificar y concretar los principios de la Economía Social en las mutualidades que operan en Euskadi, y principalmente, en su variante más importante: las Entidades de Previsión Social Voluntaria. No obstante, previamente, se analizará el significado que presenta el concepto de mutualidad, ante la ausencia de concreción en la Ley 5/2011, de 29 de marzo, de Economía Social, pese a referirse a esta como entidad de la Economía Social.

Palabras clave: Economía Social; mutualidades; Entidades de Previsión Social Voluntaria; mutuas de seguro; Euskadi.

Abstract: This paper aims to identify and specify the principles of the Social Economy within the mutual insurance organizations that operate in the Basque Country and, primarily, regarding their most important variation: the Voluntary Social Welfare Entities. Nevertheless, previously, the scope of the concept of mutual insurance organization will be analysed, due to the lack of its precision in the 5/2011 Law, of 29 March, on Social Economy, although it refers to it as an entity of the Social Economy.

Keywords: Social Economy; mutual benefit societies; voluntary social welfare entities; mutual insurance organizations; Basque Country.

Claves Econlit: K39, L53, M14.

\& Este artículo es parte del resultado de la investigación desarrollada dentro del proyecto «Construcción del relato de la Economía Social (Neurtzen II)», financiado por la Dirección de Economía Social del Departamento de Trabajo y Justicia del Gobierno Vasco durante 2019.

* Correspondencia a/Corresponding author: Francisco Javier Arrieta Idiakez. Universidad de Deusto - javier.arrieta@deusto.es

Cómo citar/How to cite: Arrieta Idiakez; Francisco Javier; Martínez Etxeberria, Gonzalo; López Rodríguez, Josune (2020). "Las mutualidades en Euskadi como entidades de la economía social», GIZAEKOA - Revista Vasca de Economía Social, 17. 181-196. (https://doi.org/10.1387/gizaekoa.22231).

Recibido: 03/06/2020; aceptado: 17/06/2020

ISSN 1698-7446 - elSSN 2444-3107 / (C) 2020 UPV/EHU

(c) (†) Esta obra está bajo una licencia

Creative Commons Atribución 4.0 Internacional 


\section{Concreción de lo que debe entenderse por mutualidad}

La Ley 5/2011, de 29 de marzo, de Economía Social, reconoce expresamente la naturaleza de entidad de la Economía Social a las mutualidades (cfr. artículo 5.1). Partiendo de dicho reconocimiento, y de la falta de concreción en dicha norma de lo que debe entenderse por mutualidades, debe matizarse que deben entenderse como tales tanto las Mutuas de seguros como las Mutualidades de Previsión Social (Paniagua Zurera, 2011, p. 195; Paz Canalejo, 2012, p. 102; Arrieta Idiakez, 2014, p. 43).

Ahora bien, la interpretación conjunta de los artículos 2, 4 y 5 de dicha norma permite concluir que, tal y como ocurre con el resto de entidades reconocidas como de la Economía Social, para que efectivamente, en la práctica, y a todos los efectos, sean consideradas entidades de la Economía Social, es preciso que cumplan con los principios orientadores de la Economía Social, así como con los requisitos consistentes, por una parte, en desarrollar actividad económica y empresarial y, por otra parte, en satisfacer bien el interés colectivo de sus integrantes, bien el interés general económico y social, o ambos (Arrieta Idiakez, 2014, p. 40).

Pero para poder averiguar si las Mutuas de Seguros y las Mutualidades de Previsión Social cumplen con dichos principios y requisitos, previamente es necesario contextualizarlas, identificarlas y definirlas.

Así, en el ámbito de la Comunidad Autónoma de Euskadi, conviene comenzar refiriéndose a la distribución competencial que opera sobre ambas formas de mutualismo, por la importancia que ello tiene para el propósito que se acaba de señalar.

De este modo, mientras que al Estado le corresponde la competencia exclusiva en materia de bases de ordenación de los seguros (cfr. artículo 149.1.11 de la Constitución) — lo que ha permitido atribuir a la Comunidad Autónoma de Euskadi la competencia de desarrollo legislativo en materia de ordenación de seguros (cfr. artículo 11.2.a del Estatuto de Gernika, Ley Orgánica 3/1979, de 18 de diciembre)—, a la Comunidad Autónoma de Euskadi le corresponde la competencia exclusiva en materia de mutualidades no integradas en la Seguridad Social (cfr. artículo 10.23 del Estatuto de Gernika).

Sin perjuicio de la conflictividad histórica existente a partir de la Ley 33/1984, de 2 de agosto, sobre ordenación del seguro privado, respecto al alcance y efectos de la competencia exclusiva del Estado en materia de bases de ordenación de los seguros en relación con la materia de mutualidades no integradas en la Seguridad Social (cfr., SSTC 97/2014, de 12 de junio; 215/2012, de 14 de noviembre; 173/2005, de 23 de junio; 35/1992, de 23 de marzo; 86/1989, de 11 de mayo), es posible definir am- 
bas formas de mutualidad conforme a las normas promulgadas con fundamento en las dos competencias exclusivas aludidas.

Así, las Mutuas de Seguro se definen conforme a lo establecido en los artículos 9 y 10 del Real Decreto Legislativo 6/2004, de 29 de octubre, por el que se aprueba el texto refundido de la Ley de ordenación y supervisión de los seguros privados, como «entidad aseguradora privada de carácter social, específica del ámbito asegurador, que se caracteriza por su carencia de ánimo de lucro y por el carácter reflexivo de su cobertura, que se dirige a sus socios, personas físicas o jurídicas» (Diccionario del Español Jurídico, 2016).

En lo que respecta a las Mutualidades de Previsión Social, en el caso de la Comunidad Autónoma de Euskadi, la particularidad reside en que las mismas se articulan por medio de las denominadas Entidades de Previsión Social Voluntaria (EPSV).

El origen de dicha particularidad lo constituye la Ley 25/1983, de 27 de octubre, que reguló por vez primera las EPSV.

En efecto, en su Exposición de Motivos se señala que «se sustituye el concepto de Mutualidad por el de Entidad de Previsión Social Voluntaria en un afán englobador, que no de uniformar, de las múltiples experiencias e instituciones de diversa índole cuyo fin último es, sin ánimo de lucro, proteger a los asociados frente a eventos que puedan poner en peligro su vida, recursos o actividad».

En ese sentido, se ha afirmado que con ello «se aprecia una sensibilidad y respeto especial hacia figuras que habían venido prestando una impagable cobertura social ante necesidades de primer orden (hermandades de asistencia funeraria que igualaban a los ciudadanos de una comunidad, villa o localidad en su último viaje, Sutearok, Basoaroak, que potenciaban el trabajo comunitario para atender los daños en los bienes raíces necesarios para la pervivencia de una sociedad agraria, montepíos de asistencia sanitaria...)» (IzQUIERDO, 2014, p. 121).

Ciertamente, las EPSV entroncan con lo que puede denominarse el espíritu comunitarista del pueblo vasco, plasmado en instituciones típicas del país, auténticas sociedades civiles constituidas bajo algunas de las formas tradicionales de cofradías, hermandades o mutualidades, y que existen desde Edad Media.

En realidad, todas estas instituciones son la proyección de una forma de entender y sentir la vida, que se encuentra condicionada por la orografía del país y fuertemente influenciada por el pensamiento cristiano.

Según se avance en organización social, se desarrollarán distintas formas de protección social.

Es así como la incipiente protección otorgada por el Derecho foral a través de las instituciones de la familia y las sucesiones (UnAmuno, 1902, 
p. 43 y ss.; Arrieta Idiakez y Lopéz Rodríguez, 20i2, 194-198), dejó paso a nuevas formas de solidaridad social, como los aprovechamientos comunes de los montes (Vicario y De la Peña, 1901, p. 92-106; Unamuno, 1902 p. 50 y ss.), las prestaciones mutuas de trabajo a trueque o trabajo en auzolan (UnAmuno, 1902, p. 54) o las aportaciones de cosas o animales denominadas lorrak (UnAmUNO, 1902, p. 55-56).

Así es como se llegó a formas de asociación ya plenamente mutualistas como las hermandades contra incendios o también denominadas sociedades de seguros mutuos contra incendios (Vicario y De la Peña, 1901, pp. 234-245), las hermandades de seguro mutuo sobre el ganado o contra los riesgos del ganado (Vicario y De la Peña, 1901, pp. 246-259; UnAMUNO, 1902, pp. 56-63), las cofradías de pescadores o mareantes (VICARIO y De la Peña, 1901, pp. 260-312) y las asociaciones mutuas de carácter funerario (Vicario y De la PeÑa, 1901, pp. 314-317).

Todas estas instituciones mutualistas se rigieron por sus propias normas, en régimen de autogobernanza y autogestión. En las provincias de Álava, Bizkaia y Gipuzkoa subsistieron incluso tras la abolición de los gremios en 1836. La promulgación de la Ley de Asociaciones de 1887 extendió su aplicación a las mismas, al referirse expresamente a los gremios, Sociedades de socorros mutuos y a las Sociedades de previsión.

No obstante, con la regulación de los seguros sociales en España, como antecedentes directos del Sistema de Seguridad Social, comenzó también a preverse una regulación diferenciada del mutualismo, con una atribución de competencias distinta respecto a ambas materias entre el Estado y las regiones autónomas.

De este modo, si la Constitución Española de 1931 otorgaba al Estado español la legislación sobre el régimen de seguros generales y sociales, sin perjuicio de que pudiera corresponder a las regiones autónomas la ejecución de dicha legislación, en la medida de su capacidad política, a juicio de las Cortes (cfr. artículo 15.8), el Estatuto Vasco de 1936, en virtud de lo dispuesto en los artículos 16 y 17 de la Constitución Española, establecía que correspondía a la competencia del País Vasco la legislación exclusiva y la ejecución directa de mutualidades (cfr. artículo 2. e).

Durante el régimen franquista, las instituciones tradicionales fueron reguladas por el Estado.

Por una parte, la protección social de los asociados a las Cofradías, que ya había comenzado a estatalizarse con la regulación en 1919 del seguro social de accidentes de mar (ARrieta Idiakez, 2007, pp. 71-79), siguió protegiéndose, primero, por los diversos seguros sociales, las mutualidades laborales y las diversas instituciones mutualistas de naturaleza especial (Arrieta Idiakez, 2007, pp. 119-130), y, después, por el Sistema de Seguridad Social (ARrieta IDIAKEZ, 2007, pp. 151-158). 
Por otra parte, la Ley de Mutualidades de 1941 se encargó de regular la previsión ejercitada con "espíritu eminentemente social y privado, para lo cual las entidades que la interpretan adoptan siempre la forma mutualista o de asociación civil» (cfr. Exposición de Motivos). El artículo 1 de esta norma no dejaba duda de que incluía en su ámbito de aplicación a las hermandades y a las asociaciones mutuas de carácter funerario, e incluso a las propias cofradías de pescadores en caso de querer estas complementar su previsión social obligatoria con la previsión social privada y voluntaria. De acuerdo con ese precepto, «se considerarán mutualidades o montepíos, a los efectos de la presente Ley, las asociaciones que con aquella denominación o con cualquiera otra, y sin ánimo de lucro, ejercen una modalidad de previsión de carácter social o benéfico, encaminada a proteger a sus asociados o a sus bienes contra circunstancias o acontecimientos de carácter fortuito y previsible a los que están expuestos mediante aportaciones directas de los asociados o procedentes de otras entidades o personas protectoras». Sin embargo, se establecía que dichas asociaciones debían regirse por sus propios Estatutos o Reglamentos ajustados a la legislación vigente, previa aprobación por el Ministerio de Trabajo (cfr. artículo 2). Es así como perduraron.

Con la instauración de la democracia, la Constitución Española de 1978 se limita a establecer en su artículo 41, in fine, que la asistencia y prestaciones complementarias al Sistema de Seguridad Social serán libres.

En cambio, el Estatuto del País Vasco de 1979, como se ha adelantado, atribuye competencia exclusiva a la Comunidad Autónoma de Euskadi en materia de mutualidades no integradas en la Seguridad Social.

Precisamente, con amparo en dicho título competencial, se aprobó la ya citada Ley 25/1983, de 27 de octubre, sobre Entidades de Previsión Social Voluntarias.

La singularidad de esas instituciones que dan lugar a las actuales EPSV se plasma también en la vigente Ley 5/2015, de 25 de junio, de Derecho Civil Vasco.

$\mathrm{Al}$ referirse a sus principios inspiradores (cfr. Capítulo II, Título preliminar), tras afirmar que «la concepción vasca de la propiedad es modulada por la función social de la propiedad y por el principio de solidaridad», establece que «las leyes ampararán las diversas formas de propiedad comunal, familiar y social peculiares del Derecho civil vasco de forma que las mismas se acomoden a la realidad social del tiempo en que deban ser aplicadas».

$\mathrm{Al}$ regular los principios del derecho patrimonial (cfr. Título I), se hace referencia a las «sociedades civiles constituidas bajo alguna de las formas tradicionales de cofradías, hermandades o mutualidades» para someterlas a la propia Ley $5 / 2015$ y determinar que las mismas se regularán por sus propios Estatutos y normas internas, siempre y cuando su contenido no se 
oponga a la Ley 5/2015, a las normas que se dicten para su desarrollo y a la legislación supletoria (cfr. artículo 16).

Con dichos antecedentes como referentes, en la actualidad, la Ley 5/2012, de 23 de febrero, sobre Entidades de Previsión Social Voluntaria (EPSV), define las EPSV como «aquellas instituciones que, sin ánimo de lucro, realizan una actividad previsora dirigida al otorgamiento de la correspondiente cobertura, en favor de sus socios ordinarios y personas beneficiarias, para las contingencias establecidas en esta ley». En concreto, deben diferenciarse las EPSV destinadas a la cobertura de contingencias personales (cfr. artículo 24) y las EPSV destinadas a la cobertura o protección de bienes del asociado (cfr. artículo 25). Entre estas últimas deben destacarse las EPSV de decesos y las EPSV de incendios (ETXEBARRIA ETXeita, 2006, pp. 76-77). Y atendiendo a la naturaleza y al vínculo entre sus socios, cabe diferenciar las siguientes modalidades de EPSV (cfr. artículo 7.b):

a) Individuales. Son aquellas cuyos socios promotores o protectores sean entidades financieras, que desarrollen las actividades de cobertura de contingencias personales, recogidas en la Ley 5/2012, para los socios ordinarios integrados en las mismas que sean personas físicas, sin que entre los mismos exista un vínculo previo que sea la causa determinante decisoria de su incorporación a aquellas entidades.

b) De modalidad de empleo. Son aquellas cuyos miembros mantengan o hayan mantenido con el socio protector una relación laboral, o de servicio en el caso del personal funcionario y estatutario, o sean socios trabajadores o de trabajo en el ámbito de las sociedades cooperativas y laborales, habiendo sido causa determinante decisoria de aquella incorporación los acuerdos alcanzados en negociación colectiva, pacto de empresa o decisión unilateral del empleador.

Asimismo, tendrán la consideración de modalidad de empleo aquellas entidades de previsión social voluntaria cuyos socios ordinarios mantengan entre sí alguna vinculación relacionada con el ejercicio de su profesión.

c) Asociadas. Son aquellas cuyos miembros mantengan entre sí una vinculación asociativa no laboral ni relacionada con el ejercicio profesional.

d) Indiferenciadas. Son aquellas cuyos miembros tengan vínculos no laborales y desarrollan actividades de cobertura de contingencias no personales.

Doctrinalmente se han definido las EPSV como «aquellas Entidades o instituciones independientes domiciliadas en Euskadi que, con carácter mutualista, se constituyen con el fin de ofertar, sin ánimo de lucro, una 
fórmula de previsión de naturaleza social y voluntaria distinta de los sistemas de previsión pública, encaminada a proteger a los miembros que la integren o sus bienes, contra circunstancias o acontecimientos fortuitos y/o previsibles» (Manual de Previsión Social Complementaria, 2009, p. 24).

Sea como fuere, es posible resaltar una serie de valores que pueden ser considerados como básicos del movimiento mutualista, en general, entre los que destacan los siguientes: la igualdad y la democracia; la libertad de participación; la justicia en la distribución de la riqueza generada o equidad; la responsabilidad de los socios por sus propios actos y por la actividad de la mutualidad; y la solidaridad (Moreno Ruiz, 2000, pp. 51 y 59).

Más concretamente respecto a las Mutualidades de Previsión Social, representadas en la Comunidad Autónoma de Euskadi por las EPSV, se afirma que asumen plenamente los principios generales que inspiran el comportamiento de las entidades de la Economía Social, entre los que cabe destacar la finalidad de servicio a los miembros más que la obtención de beneficios, la autonomía de gestión, los procesos de decisión democrática y primacía de las personas y del trabajo sobre el capital en el reparto de beneficios. Aunque también se indica que tales principios tienen una lectura singular en el caso de estas mutualidades, debido a la función social que desempeñan y a la especificidad de las actividades que desarrollan, de forma y manera que la esencia del mutualismo se traduce en dos grandes principios que inspiran el normal comportamiento de las entidades: la ausencia de ánimo de lucro y la democracia, autogestión y solidaridad (SÁEz Fernández, F.J.; Sánchez Martínez, M.T.; López Castellano, F. 2006, pp. 55-57 y Calvo Vérgez, J., 2009, pp. 64-66).

Concretamente, respecto a las EPSV se ha subrayado que las características que las distinguen respecto a otros sistemas complementarios al Sistema Público de Seguridad Social son las siguientes (Diagnóstico y Plan de Previsión Social Complementaria en Euskadi, 2007, p. 74; Urrutia y Manrique, 2011, p. 184):

a) Una finalidad concreta de previsión social que comporta, por tanto, la protección frente a contingencias sociales.

b) Los intereses de los socios de número están coordinados a través de la asociación. Ello supone un considerable grado de solidaridad.

c) Inexistencia de limitaciones para el acceso de los socios, salvo las relacionadas con los fines de la entidad.

d) Igualdad de derechos y obligaciones de los socios en relación con las aportaciones efectuadas, lo que, junto a la exigencia de una estructura y composición democrática de sus órganos de gobierno, asamblea general y junta de gobierno, produce y comporta una auténtica autogestión. La participación de los asociados en el devenir 
de su entidad de previsión les implica, en una mejor gestión y en el control de los gatos en la misma.

e) Ausencia de ánimo de lucro, tanto en la EPSV como en los participantes en los órganos de gobierno.

En todo caso, en la Comunidad Autónoma de Euskadi, son las EPSV las que destacan en número y por su importancia frente a las Mutuas de Seguros. Motivo por el que en los siguientes apartados se centrará la atención única y exclusivamente en las EPSV.

Con todo, conforme a la información facilitada por la Dirección General de Seguros y Fondos de Pensiones, entre las Mutuas de Seguros que operan en la Comunidad Autónoma de Euskadi, pueden destacarse las siguientes: (a) PAKEA, Mutualidad de Seguros a prima fija; (b) ASEMASMutua de Seguros y Reaseguros a prima (con derrama pasiva); (c) $\mathrm{Mu}$ tua de Riesgo Marítimo, Sociedad de Seguros a prima fija (MURIMAR); (d) Sociedad de Seguros Mutuos contra incendios de edificios rurales de Álava, Mutua de Seguros a prima fija; (e) SURNE, Mutua de Seguros y Reaseguros a prima fija.

Ello no obstante, sería deseable ofrecer un listado actualizado de todas las Mutuas de Seguros que operan en la Comunidad Autónoma de Euskadi, con el fin de poder valorar mejor su peso en términos económicos y sociales.

\section{Identificación y concreción práctica de los principios de la Economía Social en las mutualidades}

La Ley 5/2012, de 23 de febrero, sobre Entidades de Previsión Social Voluntaria (EPSV) contempla, en su artículo 2, los siguientes principios informadores del régimen de previsión social voluntaria, a los que deben ajustarse en su constitución y funcionamiento las EPSV:

a) Igualdad de derechos y obligaciones de todas las personas asociadas, sin perjuicio de que las aportaciones y prestaciones tengan la relación que los estatutos establezcan según las circunstancias que concurran en cada una de ellas.

b) Ausencia de ánimo de lucro, no resultando posible ni el reparto de dividendos o entrega que encubran un negocio mercantil simulado ni la retribución por la mediación de intermediarios o agentes en la incorporación de socios.

c) Transparencia en la gestión de la entidad, facilitando a los socios y a las personas beneficiarias la información necesaria de acuerdo con lo establecido en la normativa vigente. 
d) Estructura y composición democrática de sus órganos de gobierno, determinándose que la elección de esos órganos sea representativa del colectivo social, y que sus miembros tengan acceso a la información necesaria para el ejercicio de sus funciones.

e) Gratuidad en el desempeño de las funciones correspondientes a la participación en los órganos de gobierno, sin perjuicio de que, si se realizan realmente funciones ejecutivas y está previsto en los estatutos de la entidad, pueda obtenerse la aprobación de la asamblea para abonar retribuciones.

f) Inexistencia de limitación alguna para el acceso a la condición de socio o socia, de acuerdo con lo que establezcan los propios estatutos de la entidad en concordancia con sus fines.

g) Eficacia, eficiencia e innovación en la gestión de sus recursos.

Precisamente, la doctrina científica ha manifestado que esos principios se están refiriendo sin duda alguna a la Economía Social, coincidiendo en lo básico con lo que la Ley 5/2011 determina en su artículo 4 (PÉREZ URALDE, 2012, p. 88).

Por consiguiente, a continuación, se tratará de agrupar dichos principios y comentarlos, atendiendo a la clasificación realizada en función de los siguientes aspectos: centralidad de las personas y del fin social; dimensión económica y empresarial; y compromiso con la comunidad en la que se entroncan. Además, tales principios se enriquecerán con otra serie de aspectos que cabe deducir de la normativa sobre EPSV.

\subsection{Centralidad de las personas y del fin social}

La centralidad de las personas se identifica, de entrada, con el principio de gratuidad o ausencia de ánimo de lucro. No en vano, «este principio se desprende del propio carácter de las Mutualidades como asociaciones de personas que, como tales, no pretenden obtener beneficios económicos con el desarrollo de su actividad, sino alcanzar el fin social de la entidad, que es la protección social de sus miembros mediante la ayuda mutua y la solidaridad; de ahí su estructura personalista y no capitalista" (CABeza Pereiro, FERNÁNDEZ Prieto et al., 2017, p. 97).

Además, todo ello trae consigo la inexistencia de limitación alguna para el acceso a la condición de socio o socia.

En ese sentido, se afirma que «la ausencia de ánimo de lucro se da tanto en la Mutualidad, que no trata de obtener ningún beneficio repartible con su actuación, como en los mutualistas, que actúan movidos, junto a su legítimo interés personal y familiar, con un ánimo colectivo de ayuda mutua» (Congreso Español de Mutualidades, 1991, p. 111). 
En último término, "los excedentes que puedan alcanzarse con una gestión óptima de las cuotas, así como de las eventualidades aseguradas, no serán objeto de reparto, sino que se destinarán a restituir las aportaciones realizadas por los mutualistas o a la mejora de las prestaciones» (CABEZA Pereiro, Fernández Prieto et al., 2017, p. 98).

Igualmente, debe destacarse el carácter independiente de las EPSV, ya que cumplidos los requisitos específicos para su constitución disponen de personalidad jurídica propia y diferente a la de los socios (personas físicas o jurídicas) que las promueven.

Por ello, en principio, las EPSV no necesitarán del concurso de terceras entidades (gestoras) para la realización de su actividad. Cuestión distinta es la sujeción al control administrativo que ejerce el Gobierno Vasco (Manual de Previsión Social Complementaria, 2009, p. 24).

En virtud de dicha independencia cobra importancia el principio de participación democrática en el gobierno y gestión de las EPSV.

En verdad, la gestión de los órganos de gobierno de las EPSV se ajusta a la perfección al primer principio orientador de las entidades de la Economía Social, relativo a la primacía de las personas y del fin social sobre el capital, que se concreta, entre otros aspectos, en una gestión transparente, democrática y participativa (cfr. artículo 4.a de la Ley 5/2011).

Además, al tratarse de una entidad personalista, su gobierno y administración se basan en la autogestión. Una autogestión que como consecuencia de la ausencia de ánimo de lucro conlleva, como norma general, la gratuidad en el desempeño de la función de los representantes de la Entidad.

Asimismo, la autogestión llevada a cabo en términos de participación democrática en el gobierno y gestión de las EPSV requiere de fuertes criterios de transparencia respecto a todos los socios y del respeto a la igualdad de derechos y obligaciones de todas las personas asociadas.

En el mismo sentido, las EPSV deben contar con procedimientos de control interno referidos a su organización y operativa, así como con procedimientos establecidos para el control de la gestión de los riesgos de las inversiones. Además, deben contar con estructuras de participación en el control de su gestión y solvencia. En todo caso, anualmente, la asamblea general debe pronunciarse sobre la idoneidad de los mecanismos de control interno establecidos por la EPSV, mediante la aprobación del informe de la auditoría externa elaborado al efecto (cfr. artículo 56.1 de la Ley 5/2012).

Del mismo modo, los estatutos de las EPSV garantizarán la participación efectiva de representantes de los planes de previsión en los órganos de gobierno, atendiendo, para fijar el número de representantes y la condición de los mismos, al volumen de su patrimonio, número de socios o cualquier otra circunstancia o característica razonables de representación (cfr. artículo 56.2 de la Ley 5/2012). 
Debe destacarse que, por mor del principio de igualdad, las EPSV individuales deben asignar, a través de la asamblea general, un defensor o defensora del asociado que, de manera profesional e independiente del socio protector o promotor, velará por los derechos de los socios ordinarios y de los beneficiarios, resolviendo las reclamaciones que voluntariamente se le sometan (cfr. artículo 57.2 de la Ley 5/2012). Estos defensores serán entidades o expertos independientes de reconocido prestigio con conocimientos económico-financieros o jurídicos en la materia, y los gastos de designación, funcionamiento y remuneración de estos defensores en ningún caso deben ser asumidos por los reclamantes, ni afectar al patrimonio afecto a los planes de previsión social (cfr. artículo 53, apartados 2 y 3 , del Decreto 203/2015).

Por su parte, la transparencia en la gestión de la EPSV, como principio informador del régimen de previsión social voluntaria (cfr. artículo 2.c de la Ley 5/2012), consiste en facilitar a los socios y a las personas beneficiarias la información necesaria.

Además, a fin de garantizar al máximo dicha transparencia, cuando se aprecien indicios de incumplimientos reiterados y graves de las normas relativas a la transparencia y protección de los asociados o de las buenas prácticas por parte de una EPSV, el Departamento de Hacienda y Economía del Gobierno Vasco adoptará las medidas que correspondan en el marco de un procedimiento de supervisión (cfr. artículo 54.4 del Decreto 203/2015).

En último término, con todo ello se refuerza el cumplimiento de los objetivos inherentes a las EPSV, a saber, por una parte, satisfacer las prestaciones económicas convenidas al colectivo afectado o incorporado, y, por otra parte, fomentar el ahorro a través de la previsión social de todos los asociados, garantizando y protegiendo los derechos (URRUTIA Elorza, 1997, p. 66).

\subsection{Dimensión económica y empresarial}

En tanto que las Mutualidades son una forma de empresa, se cumple con la exigencia establecida en el artículo 2 de la Ley 5/2011, de 29 de marzo, de Economía Social. Y, del mismo modo, la actividad empresarial desempeñada por las EPSV conduce por sí mismo a valorar su dimensión económica.

A tenor del Documento de Reflexión sobre la Previsión Social Complementaria de Euskadi, realizado por el Departamento de Hacienda y Finanzas del Gobierno Vasco, en octubre de 2016, el patrimonio que gestionan las EPSV con planes de previsión es muy importante, pues equivale 
al 35,2\% del PIB vasco. Como se subraya en dicho documento, esta ratio refleja la considerablemente mayor implantación y peso de las EPSV en la Comunidad Autónoma de Euskadi en comparación con los Fondos de pensiones en el conjunto del Estado, cuyo patrimonio representaba el 9,5\% del PIB en 2015. Ciertamente, un volumen tan elevado de activos gestionados por las EPSV les otorga un papel relevante desde el punto de vista financiero.

Y cuantitativamente también las cifras son muy significativas. Así, en 2015 había 174 EPSV con un total de 1.556 .118 socios. De las 174 EPSV 82 otorgaban prestaciones para la jubilación y otras complementarias a la Seguridad Social, y las 92 restantes cubrían otro tipo de riesgos como son: incendios, decesos, ayudas para gastos médico y otros. Asimismo, de las 82 EPSV que otorgaban prestaciones complementarias a la Seguridad Social, 43 eran de empleo, y se estructuraban como sigue: 39 integraban planes de previsión que tienen como ámbito de actuación la empresa y 4 eran sectoriales dado que sus planes de previsión abarcaban a un conjunto de empresas (una EPSV para las empresas cooperativas pertenecientes a la Corporación Mondragón - Lagun Aro-, otra para las que se acogen a determinados convenios colectivos provinciales de Gipuzkoa - Geroa- y dos EPSV para los trabajadores del sector público, una para los trabajadores de la Administración General de la Comunidad Autónoma de Euskadi —Itzarri- y otra para los de la Administración Foral y Local —Elkarkidetza-.

Igualmente, debe resaltarse la existencia de dos Federaciones de EPSV. Por una parte, la Federación de EPSV de Euskadi, que está integrada en la actualidad por cerca de 70 Entidades que voluntariamente se han incorporado a la misma y que anualmente abonan a sus asociados prestaciones por un importe superior a los 900 millones de euros. Esta federación participa en foros de ámbito territorial diferente. Así, es miembro del Consejo Vasco de la Previsión Social, representando al Sector de las EPSV, también es miembro destacado de la Confederación Española de Mutualidades de Previsión Social Voluntaria, tiene un representante en PensionsEurope, que es la asociación más importante de pensiones de Europa, y es miembro en calidad de observador de la EAPSPI, asociación europea de pensiones del sector público (https://epsv.org/la-federacion/). Y, por otra parte, está Sutearo, Federación que integra a 34 Hermandades de Bizkaia, 4 Hermandades de Gipuzkoa y 1 Hermandad de Álava, y se centra en la protección de caseríos o viviendas situadas en el ámbito rural.

En cualquier caso, el reto pasa por extender las EPSV a toda la población, como compromiso con la comunidad, como se verá en el siguiente apartado. 


\subsection{Compromiso con la comunidad en la que se entroncan} doble.

El compromiso con la comunidad en la que se entroncan las EPSV es

Por una parte, coadyuvan a la sostenibilidad del Sistema de Seguridad Social, y, con ello, a la tasa de sustitución y, en suma, a la suficiencia de las prestaciones de Seguridad Social. En particular, las EPSV de empleo constituyen un indicador de empleo de calidad y contribuyen al reparto de la riqueza generada, pues resultando ser fruto de la negociación colectiva tienen por finalidad aumentar las pensiones de los trabajadores, en tanto en cuanto las complementan.

Por otra parte, generan importantes fondos invertibles en distintos ámbitos de interés social.

En ese sentido, la normativa de las EPSV contempla importantes aspectos relativos a la Inversión Socialmente Responsable de los fondos constituidos vía EPSV. En efecto, las EPSV están comprometidas con la Inversión Socialmente Responsable (ISR). Así, se valora la integración de los factores Medio Ambientales (A), Sociales (S) y de Buena Gobernanza (G) en el análisis fundamental, tanto en la gestión interna como en la selección de los gestores externos, contribuyendo a la consecución de los Objetivos Globales para el Desarrollo Sostenible (ODS).

En concreto, en coherencia con lo antedicho, de acuerdo con el Decreto 92/2007, de 29 de mayo, por el que se regula el ejercicio de determinadas actividades de las EPSV, en el caso de determinadas EPSV existe la obligación de poner a disposición de los socios ordinarios y de las personas beneficiarias información en torno a si la EPSV tiene en cuenta consideraciones sociales, medioambientales, éticas o de gobierno corporativo en sus inversiones. Además, cuando no lo haga, debe informar sobre las razones para no hacerlo. En cambio, cuando tenga una política en este ámbito, la describirá, al menos brevemente, especificando sus estrategias de exclusión, integración o implicación. También mencionará los activos en los que se aplica y la forma de comprobar, en su caso, la aplicación de esta política de inversión socialmente responsable (cfr. artículo 4.1.i).

Concretamente, se trata de las siguientes EPSV (cfr. artículo 1, aparta$\operatorname{dos} 1$ y 2$)$ :

a) Las que integran planes de previsión social para la jubilación, así como para fallecimiento, incapacidad permanente, desempleo de larga duración o enfermedad grave, cuyos miembros mantengan o hayan mantenido con el socio protector una vinculación laboral o sean socios trabajadores o de trabajo en el ámbito de las sociedades cooperativas y laborales (modalidad de empleo), o que, en su de- 
fecto, cotizando a cualquier Régimen de Seguridad Social o mutualidades alternativas del sistema público, mantengan entre sí una vinculación profesional o asociativa (modalidad asociada).

b) Cualquier EPSV cuya socio protector o promotor sea una entidad financiera que desarrolle las actividades de cobertura descritas en la letra anterior, para socios ordinarios y beneficiarios integrados en la misma que sean personas físicas (modalidad individual).

Asimismo, la posibilidad de que existan EPSVs con planes de previsión social a favor de personas con un determinado grado de discapacidad física o sensorial, así como de personas con discapacidad que tengan una incapacidad declarada judicialmente con independencia de su grado (cfr. Disposición Adicional tercera de la Ley 5/2012), entronca a la perfección con el tercer principio orientador de las entidades de la Economía Social, relativo a la promoción interna y con la sociedad que favorezca el compromiso con el desarrollo local, la igualdad de oportunidades entre hombres y mujeres, la cohesión social, la inserción de personas en riesgo de exclusión social, la generación de empleo estable y de calidad, la conciliación de la vida personal, familiar y laboral y la sostenibilidad.

Además, se permite realizar aportaciones a tales planes, tanto a la propia persona con discapacidad como a las personas que tengan con la misma una relación de parentesco en línea directa o colateral hasta el tercer grado inclusive, así como al cónyuge o aquellos que las tuviesen a su cargo en régimen de tutela o acogimiento (cfr. artículo 4.c de la Ley 5/2011).

Con todo, actualmente, constituyen retos la generación de empleo o la protección social de las personas cuidadoras no profesionales de familiares dependientes.

\section{Conclusiones}

Primera. Las Mutualidades forman parte de la Economía Social conforme a la Ley 5/2011, de 29 de marzo, de Economía Social, debiéndose entender que entre las mismas se incluyen tanto las Mutuas de Seguros como las Mutualidades de Previsión Social.

Segunda. En la Comunidad Autónoma de Euskadi las Mutualidades de Previsión Social se denominan y regulan como Entidades de Previsión Social Voluntaria en la Ley 5/2012, de 23 de febrero, sobre Entidades de Previsión Social Voluntaria (EPSV).

Tercera. La Ley 5/2012, de 23 de febrero, sobre Entidades de Previsión Social Voluntaria (EPSV) establece una serie de principios informado- 
res que concuerdan con los principios informadores de la Economía Social regulados en la Ley 5/2011, de 29 de marzo, de Economía Social.

Cuarta. Las principales aportaciones de las EPSV a la comunidad en la que se aplican consisten en una mejora de las prestaciones de Seguridad Social, en la inversión socialmente responsable de sus fondos en aspectos Medio Ambientales (A), Sociales (S) y de Buena Gobernanza (G) y en la preocupación por la protección social de las personas con discapacidad.

En esa línea, actualmente, el principal reto que existe es la extensión de las EPSV entre la población. Igualmente, constituyen restos la inversión para la generación de empleo de calidad y la protección de ciertos colectivos que en la actualidad carecen de la misma.

\section{Bibliografía}

Arrieta Idiakez, F.J. (2007). La Seguridad Social de los trabajadores del mar en el Derecho español. Vitoria-Gasteiz: Servicio Central de Publicaciones del Gobierno Vasco.

Arrieta Idiakez, F.J. (2014). «Concreción de las entidades de la Economía Social». En REVESCO, núm. 116.

Arrieta Idiakez, F.J. y López Rodríguez, J. (2012). «Euskal Herriko GizarteEkintza. Iraganeko oinarrietatik etorkizuneko ahalbideetara». En Jado: Boletín de la Academia Vasca de Derecho, núm. 23.

Cabeza Pereiro, Fernández Prieto et al. (2017). Manual de Protección Social Complementaria. Madrid: Tecnos.

Calvo Vérgez, J. (2009). Mutualidades de previsión social: aspectos mercantiles y fiscales. Madrid: Dykinson.

Congreso Español de Mutualidades (1991). Barcelona.

Diagnóstico y Plan de Previsión Social Complementaria en Euskadi (2007). VitoriaGasteiz: Servicio Central de Publicaciones del Gobierno Vasco.

Documento de Reflexión sobre la Previsión Social Complementaria de Euskadi. Departamento de Hacienda y Finanzas, octubre de 2016.

Etxebarria Etxeita, I. (2006). «La evolución de la previsión social en Euskadi: situación actual y perspectivas de futuro. Las nuevas propuestas normativas y fiscales». En Boletín de Estudios Económicos, vol. LXI, núm. 187.

Izquierdo, L.C. (2014). «Evolución normativa y fiscal de la previsión social complementaria en el País Vasco». En Ekonomiaz: Revista Vasca de Economía, núm. 85.

Manual de Previsión Social Complementaria (2009). Vitoria-Gasteiz: Servicio Central de Publicaciones del Gobierno Vasco. Moreno Ruiz, R. (2000). Mutualidades, Cooperativas, Seguro y Previsión Social. Madrid: CES.Paniagua Zu- 
rera, M. (2011). Las empresas de la economía social. Más allá del comentario a la Ley 5/2011, de Economía Social. Barcelona: Marcial Pons.

Paz Canalejo, N. (2012). Comentario sistemático a la Ley 5/2011, de Economía Social. Valencia: Tirant lo Blanch.

Pérez de Uralde, J.M. (2012). «Ley 5/2011, de 29 de marzo, de Economía Social: ¿su aplicación es cosa de todos? (pregunta retórica)». En Deusto Estudios Cooperativos, núm. 1.

Sáez Fernández, F.J.; Sánchez Martínez, M.T.; López Castellano, F. (2006). El mutualismo de previsión social y los sistemas de protección complementarios. Madrid: Civitas.

Unamuno, M. (1902). "Aprovechamientos comunes; Lorra; Seguro mutuo para el ganado, etc.». En Derecho consuetudinario y economía popular de España (Costa y Martínez, J.). Barcelona: Manuel Soler.

Urrutia Elorza, J.R. (1997). Modelo Autonómico de Previsión Social Voluntaria: Pasado, Presente, Futuro. Vitoria-Gasteiz: Federación de Cajas de Ahorros VascoNavarras.

Urrutia, J.R. y Manrique, F. (2011). «Evolución y retos de la previsión social voluntaria en Euskadi». En VVAA. El Estado de bienestar en la encrucijada: nuevos retos ante la crisis global. Vitoria-Gasteiz: Federación de Cajas de Ahorros Vasco-Navarras.

Vicario y De la Peña, N. (1901). Derecho consuetudinario de Vizcaya. Madrid: Imprenta del Asilo de Huérfanos del Sagrado Corazón de Jesús. 